\section{El teatro como recurso educativo en el aula de Primaria}

\section{Theatre as an educational} resource in the primary classroom

\section{Educación y Pedagogía}

$\Rightarrow$ Recibido 15/03/2018 $\checkmark$ Aceptado 18/05/2018

\section{Resumen}

El rechazo entre iguales es un precedente al acoso escolar, y el teatro como metodología activa (García-Huidobro, 2016; Navarro, 2006) es el medio que proponemos para la inclusión educativa. El diseño de investigación fue de tipo cuasi-experimental con grupo control no equivalente con evaluación antes y después del entrenamiento. La muestra de sujetos fue de 137 escolares (52,5\% niñas y $47,4 \%$ niños) de los últimos cursos de Educación Primaria con una edad media de 10,41 años. Los instrumentos de evaluación fueron cinco: tres de carácter sociométrico, una escala de autoestima y una encuesta de elaboración ad hoc sobre cuentos clásicos. El alumnado fue entrenado en improvisación teatral de cuentos. Los resultados reflejaron que los alumnos rechazados disminuyeron en el número de rechazos y en los atributos negativos recibidos por sus iguales. Los niveles de autoestima no aumentaron de manera significativa. Destacamos dos factores del programa: los cambios de rol y la aportación de los iguales-espectadores a las improvisaciones desde la cooperación. Gracias a este nuevo contexto educativo del teatro, los alumnos rompían los vínculos habituales para establecer nuevas alianzas, que modificaban las expectativas y prejuicios establecidos hacia los diferentes alumnos, especialmente los rechazados, favoreciendo la inclusión grupal.
\end{abstract}

\section{Palabras clave}

Rechazo entre iguales - Improvisación teatral ·

Aportación de los iguales - Cambio de rol 


\section{Introducción}

Desde comienzos del siglo XXI el enfoque inclusivo ha venido a sustituir a la implantada integración escolar. El término integración se centró más en las discapacidades del alumno y buscaba la adaptación del alumno al grupo, mientras que la inclusión implica que los estudiantes de una determinada comunidad aprendan juntos, con independencia de sus condiciones personales, sociales o culturales, desde una perspectiva de equidad, cooperación y solidaridad, orientada a una educación intercultural (García, 2008) El objetivo de la inclusión, por tanto, es atender a la diversidad de los estudiantes dentro de una misma aula, contando con los apoyos adecuados para dar respuesta a la heterogeneidad y centrándose en las capacidades que presenta el sujeto. Ello contribuye a fomentar la autoestima, los logros, el respeto y el sentido de pertenencia al grupo social. Finalmente, este concepto quedó establecido en la propuesta de la 48 Reunión de la Conferencia Internacional de Educación, celebrada bajo el lema "Educación inclusiva: el camino hacia el futuro" (Renato, 2008).
En el ámbito educativo, se denomina estatus grupal el grado de aceptación y rechazo de un estudiante por su grupo de iguales. Los alumnos de un grupo-clase pueden asumir diferentes estatus clasificados en los siguientes tipos sociométricos: preferidos (aceptados), rechazados, ignorados, controvertidos y promedio (García-Bacete, Jiménez, et al., 2013; García-Bacete, Sureda y Monjas, 2010; Jiménez y Lehalle, 2012). El estudio de los rasgos definitorios de los niños rechazados refleja que en su mayoría muestran conductas disruptivas o sumisas (Bierman, 2004; Cillessen, 2008). Este tipo de comportamientos disruptivos manifiesta que son niños marginados por los compañeros como consecuencia, principalmente, de sus conductas agresivas. Además, son estudiantes que no siguen las normas, no toleran las frustraciones y actúan impulsivamente, son inestables, con escasa capacidad de concentración y con baja autoestima (Bierman, 2004; Díaz-Aguado, 2004; García-Bacete, 2008). wards the different students, especially those rejected, favoring group inclusion

\section{Key Words}

Peer rejection - Theatrical improvisation - Contribution of equals · role change 
Desde el análisis de diferentes programas de intervención dirigidos a la prevención y la atención del rechazo y el acoso escolar se han reconocido como elementos favorecedores de la inclusión social los siguientes factores: trabajar desde la cooperación (Díaz-Aguado, 2006), favorecer el intercambio de estatus (Díaz-Aguado, 2002b; Guillén, 2009), implicar y concienciar al grupo de espectadores para prevenir y evitar el acoso escolar (Salmivalli, Kärnä y Poskiparta, 2011) y trabajar en equipo atendiendo a todo el contexto social (García-Bacete, Jiménez, et al., 2013). En base a este aporte, y teniendo en cuenta que desde la perspectiva inclusiva se trabaja hacia la equidad, la cooperación y la solidaridad a nivel grupa (García, 2008; Schneider, 2008), destacamos diferentes componentes del teatro implicados en dichos factores como son: el intercambio de roles, el ensayo de conductas desde la irrealidad y la aportación grupal. Se ha podido observar que estos elementos teatrales permiten mejorar la

$\frac{\infty}{0}$ inclusión gracias al fortalecimiento de la () comunicación entre los compañeros al facilitar expresarse conforme a sus posibilidades independientemente de sus capacidades (Guillén, 2009; Quirante y Curiel-Marín, 2014).
En relación con la importancia de los iguales para el desarrollo del niño, Bierman (2004) considera que los niños aceptados se sienten seguros y vinculados con los iguales en base a sus experiencias interpersonales positivas (ofrecidas en la práctica teatral), de manera que se incrementa su confianza para acercarse a los entornos sociales, además de emplear estrategias adaptativas. Por el contrario, los niños que vivencian experiencias interpersonales negativas pueden iniciarse en un ciclo recurrente de situaciones crónicas con un impacto negativo en el desarrollo de su identidad, autoestima, competencia social, salud mental y adaptación escolar.

La hegemonía de carácter cientíico-académico del sistema educativo impide que el lenguaje artístico ocupe un espacio importante en dicha institución. Sin embargo, los lenguajes artísticos como los que se trabajan con el teatro constituyen herramientas de gran valor para el desarrollo de habilidades personales, sociales y emocionales en los estudiantes (García-Huidobro, 2004; Motos, 2014; Robles y Civila, 2010). En este sentido, los autores que han utilizado el teatro como metodología educativa coinciden en afirmar que aporta a los niños vivencias de distintos sucesos simulados que fortalecen su personalidad, por ello defienden y muestran la aportación del teatro al desarrollo integral de la persona (García-Huidobro, 2016; Guillén, 2009; Motos 2014; Onieva, 2011; Orozco, 2012; Porto y Kafrouni, 2013; Robles y Civila, 2010; Tejerina, 2005; Vieites, 2014). Otras aportacio nes hacen referencia a la funcionalidad del teatro en el ámbito de la competencia social y la inclusión (Díaz-Aguado, 2004 Guillén, 2009; Henche, 2009; Morán, Ruiz y Marín, 2016; Motos, 2009; Palacios, 2009; Quirante y Curiel-Marín, 2014). La práctica teatral también se ha destacado por su aportación a la mejora de la autoestima (Alcántara, 2005; Bruner, 2004; Esteban 2009; Ferkany, 2008; Navarro, 2009; Onieva, 2011; Rocha, Levitan, Piana, Velarde Vieira, 2011) y a la creatividad (Laferrière y Motos, 2003; Oltra, 2015; Raya, 2015).

Dentro del ámbito de las artes escénicas, en esta investigación utilizamos una modalidad teatral con autonomía propia, denominada teatro de la improvisación que se caracteriza por una mayor libertad de acción sin guion predefinido. La improvisación teatral se convierte en un fin en sí mismo, que incluye al público y toma su importancia como producto y no como proceso (Renaudin, 2012; Romero y Merchán, 2008). La improvisación, por tanto, sería una representación sincera llevada a cabo por unos personajes en un espacio determinado que posibilita la expresión espontánea, liberadora y que facilita el vínculo con los otros (Merchán 2011). Desde este enfoque, el teatro de la improvisación a través de cuentos o historias permite asumir los diferentes roles o personajes dirigidos al aprendizaje del alumnado en la resolución de conflictos. Esta metodología también favorece las relaciones sociales y la tolerancia en la escuela, lo que implica favorecer la inclusión en el aula (Díaz-Aguado, 2004; Guillén, 2009). El teatro improvisado proporciona un contexto protegido para el ensayo conductual de los conflictos evitando el riesgo que supone la realidad social, porque en el contexto teatral las relaciones interpersonales tienen un mayor grado de armonía y de participación colectiva dirigidos a cumplir objetivos comunes (Guillén, 2009; Motos, 2009)

En esta investigación el cuento fue otro elemento central, que como producción literaria contribuye al desarrollo del lenguaje, a la creación literaria y a la imaginación (Alabart y Martínez, 2016; Alías, 2010; Sandoval, 2005). El cuento facilita la comunicación del niño a través del 
lenguaje simbólico, con el que va aprendiendo y asimilando conceptos sociales y morales. El cuento infantil, por tanto, ayuda a construir sólidas estructuras en la fantasía del niño y proporciona un mapa afectivo del mundo que permite un mejor entendimiento de las complejidades humanas desde el lenguaje simbólico (Rodari, 2006). Para ello, el niño fantasea y se identifica con los personajes del cuento, con sus peripecias y busca soluciones alternativas a las dificultades, permitiéndole paralelamente trabajar con los conflictos de su mundo interno. El optimismo matizado del final feliz, característico de los cuentos, permite a su vez generar expectativas positivas en los niños sobre la resolución de los conflictos sociales y personales (Alabart y Martínez, 2016).

Otro aspecto a destacar en los cuentos son los rasgos que definen a los personajes que tienden a ser ambivalentes y se caracterizan por ser exclusivamente buenos o malos. Esta particularidad ayula diferencia entre ambos caracteres. En base a este proceso, Henche $(2011,2009)$ muestra un recorrido evolutivo del desarrollo del niño a través de 12 cuentos clásicos, centrándose en la progresión de la maldad (la sombra) y la bondad (el viaje del héroe) reflejada en los personajes de dichos cuentos. Estos 12 cuentos, utilizados en esta investigación, establecen una línea divisoria entre el bien y el mal, que va transformándose y evolucionando de forma progresiva desde la seriación de los cuentos hasta integrar a personajes que alternan en su persona ambos caracteres. Esta línea divisoria va difuminándose hasta desaparecer y encontrar cualidades buenas y malas en el mismo personaje. Bettelheim (1995) confirma que dicha polaridad de lo bueno y lo malo ayuda al desarrollo infantil en el proceso de construcción del yo y en la resolución de aspectos conflictivos, inherentes a todo desarrollo humano.

La investigación que se presenta a continuación tuvo como principal objetivo determinar la aportación del teatro de la improvisación de cuentos a la disminución del rechazo de los alumnos por sus iguales. Paralelamente se establecieron otros dos objetivos: aumentar los niveles de autoestima de los alumnos y reducir los atributos negativos dirigidos hacia los alumnos rechazados.

\section{Metodología}

\section{Participantes}

La muestra de participantes en esta investigación estuvo formada por alumnos de $5^{\circ}$ y $6^{\circ}$ de Educación Primaria escolarizados en centros educativos de la ciudad de Segovia (España). La distribución aleatoria realizada concluyó con un grupo cuasi-experimental formado por tres grupos-clase y un grupo control formado por otros tres grupos-clase. El número de participantes de la muestra fue de 137 estudiantes, de los cuales 65 eran niños $(47,44 \%)$ y 72 eran niñas $(52,55 \%)$ La edad media del alumnado fue de 10,41 años, con una desviación típica de ,601 y un rango de 9-12 años.

\section{Diseño}

La investigación que se presenta siguió un modelo cuasi-experimental con un diseño de grupo control no equivalente con medidas antes y después de la intervención. El planteamiento metodológico de la investigación fue cuantitativo.

\section{Variables}

La investigación siguió un programa de entrenamiento en improvisación teatral denominado Psicoteatro y se planificaron actividades paralelas para el grupo control. Las variables intervinientes se describen a continuación:

Las variables independientes se operativizaron desde los tres componentes del programa de entrenamiento de improvisación teatral, que fueron: VI1: Dinámicas de grupo; VI2: Improvisaciones grupales y VI3: Relajación.

Las variables dependientes fueron: VD1: El estatus de los estudiantes categorizados en: preferidos, rechazados, ignorados, controvertidos y promedios (García-Bacete et al., 2010). La atención se centró en los alumnos rechazados a través de la variable Rechazos, evaluada a través de las pruebas sociométricas Valor Sociométrico y Cuestionario de Nominaciones. VD2: Los niveles de autoestima de los alumnos evaluados con la escala de Ariza (1997). VD3: Los atributos positivos y negativos de los alumnos, categorizados por Díaz-Aguado (2001). Concretamente, nos centramos en la variable atributos negativos que recibieron los alumnos re chazados, evaluado a través de la prueba sociométrica "Adivina quién es".

En un principio determinamos la homogeneidad del grupo control y cuasi-experimental en las variables sexo y 
edad a través de la prueba Chi-cuadrado. Con el objetivo de comprobar si había inicialmente diferencias significativas en las variables dependientes entre los grupos cuasi-experimental y control se aplicó ANOVA sobre los valores iniciales de dichas variables. Se obtuvo una alta homogeneidad entre ambos grupos, pero tuvimos que rechazar una de las dos variables atendidas para la hipótesis primera, que correspondió a la variable Valor Sociométrico por reflejarnos diferencias significativas en el pre-test entre los dos grupos.

\section{Objetivos e hipótesis}

Los objetivos planteados fueron: disminuir el rechazo que los estudiantes recibían por los compañeros, aumentar los niveles de autoestima y reducir la percepción negativa (a través de los atributos negativos) que recibían los estudiantes rechazados.

Las hipótesis derivadas de los objetivos fueron:

- H1: El rechazo del alumnado del grupo cuasi-experimental disminuirá en el post-test, frente al rechazo del alumnado del grupo control que mantendrá estabilidad en el post-test.

- H2: El alumnado del grupo cua- si-experimental aumentará su nivel de autoestima en el post-test, frente al alumnado del grupo control que mantendrá los valores iniciales de autoestima.

- H3: Los alumnos con estatus de rechazados del grupo cuasi-experimental reducirán los atributos negativos recibidos por sus iguales en el post-test, frente a la estabilidad de los atributos negativos recibidos los alumnos rechazados en el grupo control en el post-test.

\section{Instrumentos}

La recogida de información de los estudiantes del grupo control y cuasi-experimental se realizó a través de cinco pruebas de evaluación: tres pruebas sociométricas, una escala de autoestima y una encuesta sobre 12 cuentos clásicos de elaboración ad hoc.

Las pruebas sociométricas utilizadas permitieron determinar los estatus de cada uno de los alumnos definidos a partir de los trabajos de García-Bacete et al. (2010). Los estatus definidos son: preferidos (aceptados), rechazados, ignorados, controvertidos y promedio. Las tres pruebas de evaluación sociométrica fueron: 1.
Valor Sociométrico (VS). Con esta prueba los estudiantes de la clase valoraban en una escala numérica de uno a cinco a cada uno de sus compañeros. Los alumnos más rechazados eran los que obtuvieron mayor puntuación frente a los más aceptados que tenían menor puntuación. 2. Cuestionario de Nominaciones. Consiste en nombrar a los 3 compañeros con los que menos les gusta relacionarse y a los 3 compañeros por los que tienen más preferencia. Esta prueba aportaba el número de veces que los estudiantes eran nombrados como alumnos rechazados (R) y aceptados (E). A partir de los resultados del Cuestionario de Nominaciones se calcularon dos índices sociométricos: Preferencia Social, que corresponde al número de elecciones menos el número de rechazos (P.S.) y el Impacto Social, que es el número de elecciones más el número de rechazos (I.S.). Las puntuaciones obtenidas en P.S. determinaron el número de alumnos más rechazados y más aceptados desde valores percentiles. El grupo de alto estatus (aceptados o preferidos) fue el formado por los niños que puntuaron por encima del percentil 75 en P.S. y el grupo de bajo estatus (rechazados) fue el formado por los niños que puntuaron por debajo del percentil 25 en P.S., siguiendo el criterio de Díaz-Aguado (2001). 3. La prueba "Adivina quién es" permitió detectar a los alumnos que eran nombrados con atributos positivos (AP) y negativos (AN). Estas categorías opuestas fueron: el niño que más/menos amigos tiene, el niño más alegre /más triste, el niño que más/menos sabe, el niño que más/menos quiere la profesora, el niño que más ayuda y el que más molesta.

La escala de autoestima utilizada fue la elaborada por Ariza (1997). Esta escala consta de 25 afirmaciones que los estudiantes valoraban entre cuatro alternativas. La puntuación máxima en autoestima alcanzable es 100 frente al valor mínimo que es 25 . La confiabilidad de la escala fue de un valor de alfa de Cronbach de ,86, calculada con nuestros propios resultados a través del programa Statistical Package for the Social Sciencies (SPSS) v.20.0

La validez estuvo comprendida entre ,51 y,71 con un grado de significación de ,02

La encuesta de los 12 cuentos clásicos de elaboración ad hoc permitió recoger información sobre la preferencia de los estudiantes por un cuento determinado y una escena. Las escenas seleccionadas fueron las utilizadas durante las improvisaciones teatrales. Los 12 cuentos del listado clasificados por Henche (2009), 
fueron: 1. Los Siete Cabritillos y el Lobo, 2. Caperucita Roja, 3. Los Tres Cerditos, 4. La Casita de Chocolate, 5. Pulgarcito, 6. La Reina de las Abejas, 7. El Patito Feo, 8. Pinocho, 9. La Cenicienta, 10. La Bella Durmiente, 11. Blancanieves, 12. La Bella y la Bestia.

\section{Procedimiento}

El estatus de los alumnos y la selección de los Rechazados (R) se determinó con la evaluación inicial y se procedió a aplicar el programa de entrenamiento en improvisación teatral denominado Psicoteatro. Las sesiones de intervención teatral de grupo cuasi-experimental se planificaron en base a las escenas seleccionadas por dichos alumnos, mientras que las actividades del grupo control fueron comunes a todos.

El proceso en el grupo cuasi-experimental consistió en el entrenamiento en las técnicas de improvisación desde los cuentos seleccionados por los alumnos.

Las actividades se realizaron en salas diáfanas distintas a la clase habitual. Cada sesión de entrenamiento teatral constaba de cuatro fases: dinámicas grupales, improvisaciones y relajación.
Las dinámicas de grupo tenían una finalidad similar al caldeamiento inespecífico del Psicodrama (Hernández, 2013), cuyo objetivo era disminuir la tensión y facilitar el acercamiento físico y emocional del grupo desde un enfoque de sociabilidad distinto al de la clase habitual.

La fase de improvisaciones teatrales se dividía en tres momentos: selección de subgrupos, comunicación de los directores de escena y realización de las improvisaciones. La selección de los subgrupos era aleatoria facilitando así romper los lazos de amistad existentes. En el segundo momento se indicaba qué alumnos ejercerían de directores de su escena y en ese momento realizaban el reparto de personajes de su subgrupo. Estos alumnos habían sido seleccionados previamente y correspondían a los Rechazados intercalados con alumnos con estatus de aceptado y que destacaban en el atributo positivo "que más ayudan". Se dejaba unos minutos de planificación y se iniciaba el tercer momento que correspondía a la realización de las improvisaciones. El investigador era el narrador de dichas escenas con incitaciones a la cooperación e intercalando silencios para provocar al dialogo espontáneo entre los niños-actores.
Durante la narración, el investigador aplicaba el Stop de acción que consiste en detener la improvisación para recibir la participación del grupo. El Stop de acción permitía la aportación de alternativas ante las dudas o dificultades de los actores, con el objetivo de que contribuyeran con soluciones o ideas resolutorias de la escena. Con estos momentos de detención de la escena se invitaba a la verbalización de pensamientos y sentimientos del personaje representado por los niños-actores, que permitía especular sobre los posibles pensamientos de los protagonistas y sus intenciones $u$ objeti vos. El investigador siempre reforzaba las aportaciones de cooperación y planteaba al grupo la búsqueda de la solución ideal que permitiera la unificación y armonía grupal. El alumno que ejercía de director de escena decidía el final que se representaría y se ponía en escena. El investigador reforzaba positivamente a estos alumnos, realzando cualquier conducta o cualidad favorecedora de su integración, ignorando las conductas disruptivas. En todo momento se incitaba a la imaginación creativa y a la fantasía de los cuentos, donde la magia y la irrealidad social eran posibles.
Para la práctica de la relajación, los alumnos tumbados recibían las pautas básicas que inducen a la quietud y la relajación. En este estado de silencio y tranquilidad se les pedía que repasaran mentalmente las escenas representadas, especialmente en las que habían participado, invitando a la reflexión y el recuerdo sobre lo vivenciado.

Finalizadas los tres momentos se realizaba un debate final donde verbalizaran los sentimientos vivenciados durante las improvisaciones, y opinaban sobre su personaje y el de sus compañeros orientado a la búsqueda de cualidades nuevas y positivas en sus compañeros.

En los grupos control se realizaron visitas a los grupos-clase invitando al alumnado a recordar y verbalizar los cuentos de la lista de los 12 (Henche, 2009) que habían visto durante la evaluación inicial Esta actividad se realizó en la clase habitual del grupo y desde los pupitres.

Durante la evaluación final se aplicaron las mismas pruebas que en la inicial menos la encuesta sobre los 12 cuentos. 


\section{Resultados}

Los resultados aportados se dividen en dos bloques. En primer lugar, mostramos los datos obtenidos sobre la evolución del rechazo general mostrado en los grupos cuasi-experimental y control, y en segundo lugar, los resultados obtenidos en las tres hipótesis planteadas.

El rechazo general evaluado en los dos grupos se realizó a través del índice de Preferencia Social (P.S.). Los resultados reflejaron que el número de estudiantes rechazados (alumnos por debajo del percentil 25 en P.S.) disminuyó en el grupo cuasi-experimental un 13,6 \%, mientras que en el grupo control disminuyó un 3,3 \% (ver Tabla 1).

\begin{tabular}{|c|c|c|c|c|c|c|c|}
\hline \multirow{2}{*}{ GRUPO } & \multirow{2}{*}{$\mathbf{N}$} & \multicolumn{2}{|c|}{ PrE- test } & \multicolumn{2}{|c|}{ Post-Test } & \multicolumn{2}{|c|}{ DifERENCIA } \\
\hline & & NR & $\%$ & NR & $\%$ & NR & $\%$ \\
\hline Cuasi-experimental & 66 & 16 & $24,2 \%$ & 7 & $10,6 \%$ & 9 & $13,6 \%$ \\
\hline Control & 60 & 16 & $26,6 \%$ & 14 & $23,3 \%$ & 2 & $3,3 \%$ \\
\hline TOTALES (N) & 126 & 32 & $25,3 \%$ & 21 & $16,6 \%$ & & \\
\hline
\end{tabular}

Nota.: NR = número de niños rechazados.

Tabla 1. Frecuencia y porcentaje de niños rechazados en grupo control y cuasi-experimental

Estos resultados (ver Gráfica 1) reflejaron que el rechazo general que los alumnos mostraron en el grupo cuasi-experimental, entrenado en las técnicas de improvisación de cuentos, disminuyó en un porcentaje más alto que en el grupo control.

$$
\text { NIÑOS RECHAZADOS }
$$

$$
30,00 \%
$$$$
25,00 \%
$$$$
20,00 \%
$$$$
15,00 \%
$$$$
10,00 \%
$$$$
5,00 \%
$$$$
0.00 \%
$$

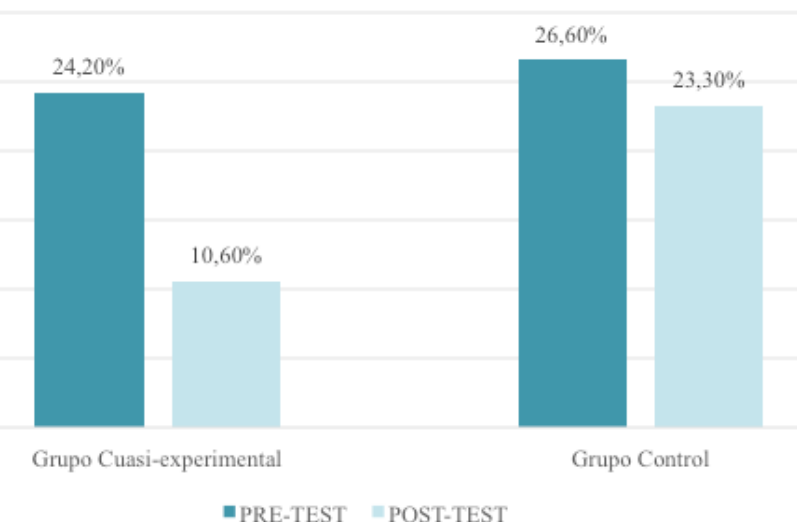

-PRE-TEST "POST-TEST
Gráfica 1. Porcentaje de niños rechazados antes y después de la intervención en ambos grupos.
Los resultados obtenidos de las tres hipótesis fueron analizados con la prueba $\mathrm{T}$ de Student. En la Tabla 2 se indican los resultados de aplicar dicha prueba junto a las medias pre-test y post-test de cada una de las variables. La hipótesis primera $(\mathrm{H} 1)$ se pudo confirmar al verificar que en el grupo cuasi-experimental las diferencias intra-grupales en la variable Rechazos ( $R)$, antes y después del entrenamiento, fueron estadísticamente significativas $(t=2,74 ; p=, 008)$, frente a las diferencias intra-grupales obtenidas en el grupo control cuyas diferencias no fueron estadísticamente significativas $(t=-, 28 ; p$ $=$,774). Con la aceptación de la $\mathrm{H} 1$ confirmamos la disminución del rechazo hacia los compañeros después del entrenamiento en las técnicas de improvisación. La hipótesis segunda $(\mathrm{H} 2)$ fue rechazada puesto que en ninguno de los grupos se obtuvo una diferencia estadísticamente significativa intra-grupalmente en los niveles de autoestima. No podemos afirmar que la práctica de Psicoteatro mejore la autoestima del alumnado. En la hipótesis tercera $(\mathrm{H} 3)$ contrastamos los atributos negativos que recibieron los estudiantes rechazados antes y después de la intervención. Los resultados reflejaron que las diferencias intra-grupales, en relación a los atributos negativos en el grupo cuasi-experimental fueron estadísticamente significativas ( $\mathrm{t}=2,18 ; \mathrm{P}=, 049)$, mientras que en el grupo control no se reflejó una diferencia significativa ( $t=, 40 ; P=, 694)$. La confirmación de la H3 permitió determinar que el entrenamiento en las técnicas de improvisación hizo disminuir el número de atributos negativos que recibían los alum-

\begin{tabular}{|c|c|c|c|c|}
\hline \multirow[b]{2}{*}{ HIPÓTESIS } & \multicolumn{2}{|c|}{ GRUPO CUASI-EXPERIMENTAL } & \multicolumn{2}{|c|}{ GRUPO CONTROL } \\
\hline & MEDIA & $\begin{array}{l}\text { CONTRASTACIÓN } \\
\text { INTRA - GRUPAL }\end{array}$ & MEDIA & $\begin{array}{l}\text { CONTRASTACIÓN } \\
\text { INTRA - GRUPAL }\end{array}$ \\
\hline $\begin{array}{l}\text { H1: } \\
\text { Rechazos }\end{array}$ & $\begin{array}{ll}\text { Pret } & 2,48 \\
\text { Post } & 1,79\end{array}$ & $(t=2,74 ; p=, 008)$ & $\begin{array}{ll}\text { Pret } & 2,36 \\
\text { Post } & 2,42\end{array}$ & $(t=-, 28 ; p=, 774)$ \\
\hline $\begin{array}{l}\text { H2: } \\
\text { Autoestima }\end{array}$ & $\begin{array}{ll}\text { Pret } & 79,13 \\
\text { Post } & 80,34 \\
\end{array}$ & $(t=1,30 ; p=, 198)$ & $\begin{array}{ll}\text { Pret } & 76,67 \\
\text { Post } & 76,15 \\
\end{array}$ & $(\mathrm{t}=, 54 ; \mathrm{p}=, 588)$ \\
\hline $\begin{array}{l}\text { H3: Atributos negativos } \\
\text { de alumnos rechazados }\end{array}$ & $\begin{array}{ll}\text { Pret } & 6,03 \\
\text { Post } & 4,12\end{array}$ & $(t=2,18 ; P=, 049)$ & $\begin{array}{ll}\text { Pret } & 6,21 \\
\text { Post } & 5,80\end{array}$ & $(t=, 40 ; P=, 694)$ \\
\hline
\end{tabular}
nos rechazados por sus compañeros en el grupo cuasi-experimental.

Nota: Pret $=$ Pre-test $;$ Post $=$ Post-test.

Tabla 2. Resultados de las hipótesis 
El análisis estadístico de los resultados obtenidos en la investigación nos permitió aceptar la $\mathrm{H} 1$ y la H3, confirmando que el entrenamiento en la improvisación teatral de cuentos disminuyó el rechazo y el número de atributos negativos que recibían los alumnos rechazados por sus compañeros. La H2 fue rechazada pues to que los niveles de autoestima no aumentaron significativas en ninguno de los grupos.

\section{Discusión y conclusiones}

De los elementos que intervienen en el teatro de la improvisación, señalamos como potenciadores de la inclusión, por una parte, la aportación de los iguales a las escenas desde la cooperación y por otra a los cambios de rol durante las simulaciones. En primer lugar, las aportaciones del grupo predisponían a los alumnos espectadores a la reflexión sobre los hechos simulados y a su participación. Esta perspectiva está en consonancia con el éxito de los programas de integración dirigidos a la prevención e intervención del acoso escolar, al plantear la necesidad de no centrarse exclusivamente en la víctima y el acosador, sino influir en los testigos del rechazo (Salmivalli, Kärnä y Poskiparta,
2011; Thompson y Smith, 2013). El objetivo de estos programas era abandonar la actitud de indiferencia que hacía cómplices del rechazo a los observadores y potenciar su implicación para que fueran capaces de empatizar, defender y apoyar a la víctima. En nuestro programa de entrenamiento, las aportaciones de los iguales hacían referencia a las alternativas resolutorias de las escenas de los cuentos buscando la máxima cooperación grupal. Se potenciaba la participación espontánea para formar parte activa de la acción, incitando al grupo a participar de manera libre y voluntaria para la resolución de las escenas, como se caracteriza al teatro de la improvisación de acuerdo con Romero y Merchán, (2008). En consecuencia, podemos aportar que la improvisación teatral potencia la implicación grupal y predispone al cambio de normas grupales orientadas a la cooperación que mejoran la inclusión de los alumnos.

En relación al cambio de rol durante las simulaciones, debemos tener en cuenta que las reglas que definen la técnica de la improvisación teatral incitan al grupo a la cooperación. Estas reglas se caracterizan por predisponer a la persona a la escucha activa y a tener un objetivo que permita concentrarse fuera de sí mismo atendiendo a las necesidades del grupo (Layton, 1990). La combinación de estas reglas permite que los alumnos aprendan a buscar objetivos comunes que potencien el beneficio del grupo y faciliten la cooperación. En nuestro estudio, estas reglas de la improvisación permitieron que los niños jugaran a actuar sin mostrar reproches, ni juzgar la intervención de los compañeros. Se generó un contexto en el que todo valía, no había representacio nes correctas o incorrectas. Únicamente tenían que aceptar las improvisaciones de los compañeros y seguirles durante la acción. Esta perspectiva de libertad durante las improvisaciones concuerda con los resultados obtenidos por Fernández, Guerra y Begara (2013), al relatar en su análisis retrospectivo de un grupo de teatro que la interpretación permitía a los pacientes expresarse sin temor a ser juzgados o rechazados, dejando de ser lo que eran para asumir otro personaje. En este sentido, el teatro de la improvisación es una metodología que facilita el intercambio de roles, pero además potencia el equilibrio grupal, gracias al Ilamado principio del Balancín definido por Johnstone (1990). Siguiendo este principio, los alumnos practicaban los distintos estatus de superioridad, inferioridad o igualdad en función del personaje a representar y a los rasgos que lo definían. En el entrenamiento de las improvisaciones de nuestro estudio se potenciaba que todos asumieran alguna vez un estatus diferente para poder trabajar desde la perspectiva de igualdad el equilibrio grupal. Este intercambio de estatus o cambio de roles podemos concluir que influyó en la modificación de las expectativas hacia los niños rechazados, facilitando la disminución de dichos rechazos. Especialmente si tenemos en cuenta que los niños más aceptados por sus compañeros se caracterizan por permitir ejercer temporalmente el control de las relaciones, intercambiar el estatus o mantener un estatus similar (Díaz-Aguado, 2002b).

La improvisación teatral de los cuentos clásicos proporcionaba una identificación con los personajes de manera más integral que con sólo la escucha de la narración, porque el lenguaje simbólico de los cuentos además de facilitar la asunción del personaje proporciona, de acuerdo con Rodari (2006), un mayor entendimiento de la complejidad afectiva del ser humano. Especialmente es efectivo cuando los protagonistas de los cuentos son niños o adolescentes que se enfrentan a peligros de la vida sin contar con la ayuda de los padres, superando los obstáculos 
que se les presentan en el camino de la vida con astucia, inteligencia, coraje, bondad, fantasía o magia; entre otras cualidades. La superación de los obstáculos se da desde el personaje que representan porque, como defiende Fernández et al. (2013), el actor aprende de sus personajes cuando despliega distintos roles o formas de ser y hace uso de habilidades, estrategias y motivaciones que caracterizan a dicho personaje.

De acuerdo con Díaz-Aguado (2004), la tolerancia grupal que se desprende de las simulaciones establece relaciones más estrechas que no suelen darse de forma espontánea desde el pupitre. En nuestra investigación pudimos observar que el acercamiento físico en los escenarios improvisados generaba nuevos vínculos entre los alumnos, porque inicialmente se rompía la estabilidad de la estructura interna del grupo y se generaba una necesidad de reestructurar nuevas alianzas socioafectivas para las representaciones.

Estas nuevas alianzas momentáneas moD dificaban las expectativas y prejuicios establecidos hacia los diferentes alumnos, que se vio reflejado en la disminución de número de atributos negativos hacia los alumnos rechazados. Dichas aportaciones estarían también en concordancia

con la afirmación de que el teatro es una metodología activa (García-Huidobro, 2004; Navarro, 2006) porque permite descontextualizar al alumno de la inmovilidad del aula-clase, trabajar desde la irrealidad y generar nuevos vínculos sociales.

Los alumnos que participaron en el entrenamiento de improvisación teatral, aunque aumentaron los niveles de autoestima fue de manera no significativa, por este motivo no podemos afirmar que la práctica teatral durante esta investigación mejoró la autoestima. Aun así, dicho resultado no concuerda con aportaciones que defienden que el teatro permite modificar la imagen que se tiene de uno mismo al expresar y transformar vivencias durante las improvisaciones y que, por tanto, mejora la autoestima desde la irrealidad de las situaciones (Esteban, 2009; Navarro, 2009; Onieva, 2011; Rocha et al., 2011).

En base a lo expuesto, podemos concluir que el programa del teatro de la improvisación de cuentos, denominado Psicoteatro, permitió aumentar los niveles de inclusión y reducir los atributos negativos dirigidos hacia los alumnos rechazados. Gracias a este nuevo contexto educativo del teatro se rompen los vínculos habi- tuales entre los alumnos para establecer nuevos, con la carga afectiva del rol del personaje representado que favorece la inclusión de todos los alumnos y fortalece el aporte cooperativo de los iguales. Consideramos que esta investigación es de gran interés, porque el teatro es una potente herramienta educativa y hasta ahora su funcionalidad ha sido principalmente artística, sin valorar el aporte al desarrollo inclusivo del alumnado.

Sin embargo, consideramos que nuestro estudio no estuvo exento de limitaciones. Con el objetivo de subsanarlas destacamos la necesidad de aumentar el tamaño de la muestra atendiendo a otras poblaciones españolas, así como aumentar el tiempo de intervención. También sería beneficioso implicar al contexto educativo de profesores y familiares en futuras investigaciones. Consideramos importante incorporar otras variables de tipo cognitivo y psicolingüístico que permitirán un registro más exhaustivo de las narraciones espontáneas de los alumnos. Las perspectivas de futuro planteadas estarían orientadas a suplir estas limitaciones y especialmente sería de gran interés atender a la formación del maestro en técnicas activas como la improvisación teatral (Vieites, 2014), que les posibilite un acercamiento más práctico al desarroIlo social, personal y emocional de los estudiantes.

En el futuro y orientado a la detección de los alumnos rechazados haríamos uso de herramientas online como el denominado socio-emociograma elaborado por De Juan y Martín (2015), que permite evaluar las competencias interpersonales y las relaciones de influencia, rechazo o conflicto, que se den en el grupo. Aporta además resultados de la integración y exclusión de los alumnos, así como de la cohesión grupal.

No obstante, consideramos que este estudio aporta datos relevantes para entender los mecanismos que subyacen al proceso de inclusión y que nos permitirá elaborar programas teatrales con la implicación de todo el contexto educativo.

\section{Agradecimientos}

Nuestro reconocimiento y agradecimiento a la Asociación Psicoteatro para la Paz, a los orientadores educativos, directores, maestros, padres y alumnos de los centros de Educación Primaria de Segovia que participaron en la investigación. 
A Tomás Motos, Irene Henche y Verónica García-Huidobro, con quienes hemos compartido impresiones y recibido grandes aportaciones. Es de destacar el apoyo recibido por Juan Carlos Manrique Arribas y Ruth Pinedo González en este trabajo. Quiero agradecer a la Universidad Internacional de la Rioja la gran labor que realiza orientando e innovando a sus profesores con numerosos cursos formativos, especialmente a Raúl Romero impulsor para publicar este artículo por su excelente y extensa aportación sobre las publicaciones de impacto.

\section{Referencias Bibliográficas}

Alabart, M. A. y Martínez, E. (2016). Educación emocional y familia. El viaje empieza en casa. Barcelona: Grao.

Alcántara, J. (2005). Educar a la Autoes tima. Barcelona: CEAC.

Alías, D. (2010). Posibilidades metodológicas del cuento motor en educación infantil y primaria: aproximación teórica. Revista on-line de la Educación en Extremadura, 5, 62-69.

American Psychological Association, $\infty$ APA (2010). Publication manual of de American Psychological Association. (6a ed.). Washington, D. C.: APA.
Ariza, J. (1997). Test de medición de la autoestima. Recuperado de http:// es.scribd.com/doc/"/ 140292745 / test-de-mediciOn-de-la-autoestima-pdf\#scribd

Bettelheim, B. (1995). Psicoanálisis de los cuentos de hadas. Barcelona: Ed. Crítica. (Versión ori. 1978)

Bierman, K. L. (2004). Peer rejection. Developmental, processes and intervention strategies [EI rechazo entre iguales. Estrategias de desarrollo, procesos e intervención]. New York: The Guilford Press.

Bruner, J. (2004). Realidad mental y mundos posibles. Barcelona, España: Romanyà/Valls. (Obra ori. 1990)
Cillessen, A. H. N. (diciembre, 2008) Peer rejection: developmental and contextual variations [EI rechazo entre iguales: desarrollo y variación contextual]. En las III Jornadas internacionales sobre recha zo entre iguales: Acoso Grupal Permanente. Grupo Grei. En Castellón del 11 al 12 de diciembre de 2008.

De Juan, J. y Martín, D. (2015). Socio-emociograma: instrumento para la evaluación Online de las competencias socio-emocionales en el aula. En AIDIPE (Ed.), Investigar con y para la sociedad (Vol. 3, pp. 1703-1715). Cádiz, España: Bubok. Recuperado de http://aidipe2015. aidipe.org

Díaz-Aguado, M. J. (2001). Programas de prevención de la violencia y la exclusión social. Madrid: Instituto de la Juventud Ministerio de Trabajo y Asuntos Sociales. Informe de investigación inédito.

Díaz-Aguado, M. J. (2002b). Educación intercultural y aprendizaje cooperativo. Madrid: Pirámide.
Díaz-Aguado, M. J. (2004). Prevención de la violencia y lucha contra la exclusión desde la adolescencia. Madrid: Instituto de Juventud, Ministerio de Trabajo y Asuntos Sociales. Tres libros y un vídeo.

Díaz-Aguado, M. J. (2006). Del acoso escolar a la cooperación en las aulas. Madrid Pearson Educación.

Esteban, M. (2009). Las ideas de Bruner: "de la revolución cognitiva" a la "revolución cultural". Educere: Revista Venezolana de Educación, 44, 235-241.

Ferkany, M. (2008). The educational importance of self-esteem [La importancia de la educación en la autoestima]. Journal of Philosophy of Education, 42, 119-132.

Fernández, M., Guerra, P. y Begara, O. (2013). El teatro como instrumento tera péutico en la rehabilitación psicosocial Norte de salud mental, 11 (46), 82-88.

García-Bacete, F. J. (2008). Identificación de subtipos sociométricos en niños y niñas de 6 a 11 años. Revista Mexicana de Psicología, 25 (2), 209-222. 
García-Bacete, F. J., Sureda, I., y Monjas, I. (2010). El rechazo entre iguales en la educación primaria: una panorámica general. Anales de Psicología, 26 (1), 123-136.

García-Bacete, F. J., Jiménez, I., Muñoz M. V., Monjas, M. I., Sureda, I., Ferrà, P.,... Sanchiz, M. L. (2013). Aulas como contextos de aceptación y apoyo para integrar a los estudiantes rechazados. Apuntes de Psicología, 31(2), 145-154.

García-Huidobro, V. (2004). Pedagogía Teatral, metodología activa en el aula. Santiago de Chile: Universidad Católica de Chile.

García-Huidobro, V. (2016). La UC impulsa la incorporación del teatro como asig natura en básica y media. Publicado en la página web de la Pontificia Universidad Católica de Chile (UC). Recuperado de https://cl.universianews.net/2016/01/11/ la-uc-impulsa-incorporacion-de-teatro-como-asignatura-en-basica-y-media/

García, J. L. (2008). Aulas inclusivas. Bordón 60 (4), 89-105.

Guillén, M. A. (2009). El teatro como estrategia psicopedagógica para favorecer la inclusión de niños preescolares. X Con- greso Nacional de Investigación Educativa (21-25 de noviembre de 2009). Recuperado de http://www.comie.org.mx/congreso/memoriaelectronica/v10/pdf/area_tematica_01/ponencias/1007-F.pdf

Henche, I. (2009). Educar en valores a través de los cuentos. Buenos Aires: Bonum.

Henche, I. (2009). El viaje del héroe a través de los doce cuentos. Anamorphosis, 7, 86-92.

Henche, I. (2011). El conflicto y la lucha contra los adversarios a través del Psicodrama Simbólico de los cuentos de hadas. Revista Vínculos del ITGP, (2ª etapa), 4, 4-13.

Hernández, S. (2013). Psicodrama con niños y adolescentes: breve introducción psicoterapéutica. Revista Digital de Medicina Psicosomática y Psicoterapia, 3(2), 1-37.

Jiménez, T. y Lehalle, H. (2012). La violencia escolar entre iguales en estudiantes populares y rechazados. Intervención Psicosocial, 21(1), 77-89.

Johnstone, K. (1990). Improvisación y el teatro. Chile: Editorial Cuatro Vientos.
Laferrière, G. y Motos, T. (2003). Palabras para la acción. Términos de teatro en la educación y la intervención sociocultural. Ciudad Real: Ñaque.

Layton, W. (1990). ¿Por Qué? Trampolín Del Actor. Madrid: Editorial Fundamentos.

Merchán, C. (2011). El espacio escénico pedagógico1: la construcción del rol docente. Folios, 33, 113-125.

Morán, J.T., Ruiz, M. E. y Marín, D. (2016). El teatro, un recurso para el desarrollo de competencias y para la inclusión. Aula de innovación educativa, 248, 39-43.

Motos, T. (2014). Psicopedagogía de la dramatización. Recuperado de http:// www.postgradoteatroeducacion.com/ wpcontent/uploads/2016/11/Psicopedagog\%C3\%ADa_Drama_MTA_2015_2016. pdf

Motos, T. (2009). El teatro en la educación secundaria: fundamentos y retos. Revista Creatividad y Sociedad, 14(1).Recuperado de http://centroderecursos alboan.org/ebooks/0000/0847/5_APY_ REE_2.pdf
Navarro, N. (2006). El valor pedagógico de la dramatización: su importancia en la formación inicial del profesorado. Creatividad y Sociedad, 9, 11-18.

Navarro, N. (2009). Drama y Educación en Inglaterra: una Mirada a través de algunos de sus protagonistas. Creatividad y Sociedad, 14. Recuperado de www.creatividady sociedad.net

Oltra, M. A. (2015). Educación intercultural, diversidad y creatividad en el aula a través del teatro. Didáctica (Lengua y literatura), 27, 167-182.

Onieva, J. L. (2011). La dramatización como recurso educativo: Estudio compa rativo de una experiencia con estudiantes malagueños de un centro escolar concertado y adolescentes puertorriqueños en situación de marginalidad (Tesis doctoral inédita). Recuperada de http://riuma.uma. es/xmlui/bitstream/handle/10630/4892/ TDR\%20"/TDR\%20ONIEVA\%20LOPEZ.pd$\mathrm{f}$ ? sequence $=1$

Orozco, Z. (2012). El teatro como estrategia didáctica para mejorar la autorregu lación de la conducta en niños con TDAH. Escenarios o10 (1), 69-82. 
Palacios, I. M. (2009). Taller de teatro en la escuela. Innovación y Experiencias Educativas, 20. Recuperado de https://archivos.csif.es/archivos/andalucia/ensenanza/revistas/csicsif/revista/pdf/Numero_20/ISABEL_PALACIOS_1.pdf

Porto, A. A. y Kafrouni, R. (2013). Teatro y desarrollo psicológico infantil. Avances en Psicología Latinoamericana, 31 (3), 575-585.

Quirante, M. R. y Curiel-Marín, E. (marzo, 2014). El teatro en la escuela: un recurso para la inclusión. Trabajo presentado en Innovagogía 2014. II Congreso Virtual Internacional sobre innovación pedagógica y praxis educativa. Recuperado de http://www. innovagogia.es/

Raya, A. (2015). Taller de creatividad para todo el centro escolar. Revista Padres y Maestros,362, 39-43.

Renato, O. (noviembre, 2008). Inclusión Educativa: El Camino del futuro un desafío para compartir. Trabajo presentado en la $48^{a}$ Conferencia Internacional de Educación (CIE 2008). Ginebra. Recuperado de http://www.ibe.unesco.org/fileadmin/user_upload/ COPs/News_documents/2007/0710PanamaCity/Documento_Inclusion_Educativa. pdf

Renaudin, J. (2012). La improvisación dramática como entrenamiento estratégico en el aula de lengua extranjera. Revista de didáctica español como lengua extranjera, 14, 1-18.

Robles, G. y Civila, D. (2010). El taller de teatro: una propuesta de educación integral. Recuperado de http://www.rieoei.org/deloslectores/642Robles.PDF

Rocha, J., Levitan, D., Piana A., Velarde, V.C., Vieira A. (2011). Teatro sin vergüenza: jóvenes, oficinas estéticas y mudanzas en las imágenes de sí mismo. Psicología: ciência eprofissao, 31 (1), 66-79.
Rodari, G. (2006). Gramática de la fantasía (6 ed.). Barcelona: Planeta.

Romero, J. H. y Merchán, A. A. (2008). Improvisación: herramienta fundamental para el actor. Revista Colombiana de las Artes Escénicas, 2 (1), 23 - 29.

Salmivalli, C., Kärnä, A., y Poskiparta, E. (2011). Counteracting bullying in Finland:The KiVa program and its effects on different forms of being bullied [Combatiendo el acoso en Finlandia: el programa KiVa y sus efectos en diferentes formas de ser acosadores]. International Journal of Behavioral Development, 35(5), 405-411.

Sandoval, C. (2005). El cuento infantil: una experiencia de lenguaje integral. Revista Electrónica de la Red de Investigación Educativa, 1(2). Recuperado de http://revista.iered. org

Schneider, B. (diciembre, 2008). La oración de la serenidad: intervenir para mejorar las relaciones entre iguales. En Las II Jornadas Internacionales Sobre Rechazo Entre Iguales: Acoso Grupal Permanente. Grupo GREI. En Castellón, España del 11 al 12 de diciembre de 2008.

Tejerina, I. (2005). El juego dramático en primaria. Alicante, España: Biblioteca Virtual Miguel de Cervantes.

Thompson, F. y Smith P. K. (2013). Estrategias antibullying dentro de los centros: lo que se hace y lo que funciona. En A. Ovejero, P.K. Smith y S. Yubero, El acoso escolar y su prevención. Perspectivas Internacionales (pp. 241-262). Madrid, España: Biblioteca Nueva.

Vieites, M. F. (2014). Educación teatral: una propuesta de sistematización. Teoría de la Educación. Revista interuniversitaria, 26(1), 36-47. 